\author{
D. J. Wexler • R. W. Grant • E. Wittenberg • \\ J. L. Bosch · E. Cagliero - L. Delahanty • \\ M. A. Blais · J. B. Meigs
}

\title{
Correlates of health-related quality of life in type 2 diabetes
}

Received: 20 October 2005 / Accepted: 6 March 2006 / Published online: 29 April 2006

(C) Springer-Verlag 2006

\begin{abstract}
Aims/hypothesis: We assessed the impact of medical comorbidities, depression, and treatment intensity on quality of life in a large primary care cohort of patients with type 2 diabetes. Methods: We used the Health Utilities Index-III, an instrument that measures healthrelated quality of life based on community preferences in units of health utility (scaled from $0=$ death to $1.0=$ perfect health), in 909 primary care patients with type 2 diabetes. Demographic and clinical correlates of health-related quality of life were assessed. Results: The median health utility score for this population was 0.70 (interquartile range $0.39-0.88$ ). In univariate analyses, older age, female sex, low socioeconomic status, cardiovascular disease, microvascular complications, congestive heart failure,
\end{abstract}

D. J. Wexler $(\bowtie) \cdot$ E. Cagliero $\cdot$ L. Delahanty

Diabetes Unit, Department of Medicine,

Massachusetts General Hospital,

Boston, MA, USA

e-mail: dwexler@partners.org

Tel.: +1-617-7268722

Fax: +1-617-7248534

R. W. Grant · J. B. Meigs

General Medicine Division, Department of Medicine,

Massachusetts General Hospital and Harvard Medical School, Boston, MA, USA

E. Wittenberg $\cdot$ J. L. Bosch

Institute for Technology Assessment, Massachusetts General Hospital and Harvard Medical School,

Boston, MA, USA

J. L. Bosch

Department of Epidemiology and Biostatistics, Erasmus MC

University Medical Centre,

CA Rotterdam, The Netherlands

\section{J. L. Bosch}

Department of Radiology, Erasmus MC

University Medical Centre,

CA Rotterdam, The Netherlands

M. A. Blais

Department of Psychiatry, Massachusetts General Hospital,

Boston, MA, USA peripheral vascular disease, chronic lung disease, depression, insulin use and number of medications correlated with decreased quality of life, while obesity, hypertension and hypercholesterolaemia did not. In multiple regression analyses, microvascular complications, heart failure and depression were most strongly related to decreased healthrelated quality of life, independently of duration of diabetes; in these models, diabetes patients with depression had a utility of 0.59 , while patients without symptomatic comorbidities did not have a significantly reduced quality of life. Treatment intensity remained a significant negative correlate of quality of life in multivariable models. Conclusions/interpretation: Patients with type 2 diabetes have a substantially decreased quality of life in association with symptomatic complications. The data suggest that treatment of depression and prevention of complications have the greatest potential to improve health-related quality of life in type 2 diabetes.

Keywords Adult diabetes - Chronic disease ·

Health-related quality of life

Abbreviations COPD: chronic obstructive pulmonary disease - HANDS: Harvard Department of Psychiatry/ National Depression Screening Day Scale · HUI-3: Health Utilities Index Mark III - UKPDS: UK Prospective Diabetes Study

\section{Introduction}

Diabetes results in life-threatening complications and reduced life expectancy, but only after many largely asymptomatic years during which patients must adhere to bothersome and difficult therapy. While the UK Prospective Diabetes Study (UKPDS) found that complications of diabetes affect quality of life more than overall treatment intensity [1], many patients find treatment itself burdensome $[2,3]$. In addition, the prevalence of depression is substantially higher among patients with diabetes than those without diabetes [4]. Depression, in turn, decreases 
quality of life [5,6] and hinders treatment [7]. While there have been a number of studies of quality of life in patients with type 2 diabetes $[1,8-12]$, few have looked at a large primary care cohort while simultaneously accounting for multiple medical comorbidities, depression and treatment intensity.

To address these questions, we conducted a survey of over 900 primary care patients with type 2 diabetes using the Health Utilities Index Mark III (HUI-3) [13-15] to measure quality of life. The HUI-3 is a questionnaire that links patients' responses about their level of function in eight different health domains to previously defined health utilities derived from the general population. We estimated the utility associated with type 2 diabetes and determined the factors that contribute to diminished health-related quality of life, including clinical comorbidities, depression and treatment. We hypothesised that symptomatic comorbidities of diabetes would account for much of the decrement in health-related quality of life associated with diabetes.

\section{Subjects and methods}

\section{Study population}

We surveyed patients with diagnosed diabetes who were followed in one of two outpatient primary care medical clinics between December 2001 and July 2003: a community health centre in Revere (MA, USA), and a hospitalbased primary care internal medicine practice in Boston (MA, USA). We generated lists of potentially eligible patients using billing claims for non-gestational diabetes (codes 250.00-250.90 in the International Classification of Diseases, 9th revision) over a 3-year period. Type 2 diabetes was confirmed by detailed chart review of the patient's medical record by trained research nurses. Diabetes was defined by adult-onset or obesity-associated diabetes diagnosis listed in the problem list, diabetesspecific medicine (e.g. sulfonylurea, metformin, insulin or equipment for insulin injection or home glucose monitoring) listed in the medication list, or diabetes diagnosis discussed in a progress note. Patients with clinical type 1 diabetes (defined by age of onset less than 40 years in conjunction with insulin treatment since diagnosis, designations of 'juvenile diabetes', or positive anti-islet cell antibodies when available) were excluded, and none was detected in the chart re-review. Eligible patients were diagnosed with diabetes before the intervention period, were alive at study completion, and received continuous care at their designated clinical site, with at least one visit in the first half and one in the second half of the study period. Patients with a terminal illness or cognitive impairment were excluded.

A total of 1,648 potential participants were mailed a letter co-signed by their primary care physican and the principal investigator (J. B. Meigs) describing the study. Of these, $18 \%$ either opted out from further contact or were initially excluded. The remainder (1,317 patients) were contacted by telephone in advance of a usual care clinic visit to arrange a meeting with a study staff member. Nine hundred and fiftythree patients $(72.4 \%)$ provided informed consent and completed the study survey. Of those who did not participate, approximately one-third declined; one-quarter either did not arrive for their appointment, promised to complete the survey at home but did not, or could not be reached; and one-quarter were either not diabetic or excluded owing to illness or inability to participate. Limited chart data available on non-participants revealed that they had the same mean age as participants (66 years, $p=0.6$ ), similar Charlson comorbidity scores (2.7 vs $2.9, p=0.06$ ), and a similar prevalence of depression ( $33 \mathrm{vs} 36 \% p=0.2)$ and prescription of antidepressants (22 vs $21 \%, p=0.7$ ). Non-participants were less likely to be white ( 72 vs $83 \%$ of participants, $p<0.0001$ ), less likely to speak English ( 87 vs $98 \%, p<0.0001$ ), more likely to be receiving Medicaid (9 vs $6 \%, p=0.04$ ), and less likely to have an $\mathrm{HbA}_{1 \mathrm{c}}<7 \%$ (39 vs 46\%, $p=0.01$ ). Non-participants had higher blood pressure and were more likely to be on an antihypertensive medication ( 61 vs $54 \%, p=0.01$ ) or aspirin (28 vs 20\%). The final study population consisted of 909 patients with type 2 diabetes. The Massachusetts General Hospital Institutional Review Board approved the study.

\section{Demographic and clinical covariates}

Demographic data were derived from survey responses. Clinical data were collected from manual chart reviews, directly from the electronic medical record, from the hospital's central data repository (laboratory test dates and results), from billing claims (hospitalisations and hospital discharge diagnoses), and from administrative records (patient demographics and insurance status).

Comorbidities were determined based on medical record review of listed diagnoses. A condition, its synonyms, and/ or its specific treatments were taken as indications of a given comorbidity. Microvascular complications were defined based on any one the following diagnoses, their synonyms, or their abbreviations on the problem list: neuropathy, amputation, foot ulcer, gastroparesis, diabetic diarrhoea, impotence, postural hypotension, nephropathy, renal failure, nephrotic syndrome, renal transplant, dialysis, microalbuminuria, renal insufficiency, retinopathy or photocoagulation. Microvascular complications were grouped because they do not occur independently, and numbers of patients with individual complications were too small to allow sufficient power to determine changes in quality of life. Because asymptomatic conditions may be less likely to affect quality of life, we also defined a subset of patients more likely to have symptomatic microvascular complications by excluding any patients identified solely by one or more potentially asymptomatic diagnoses (nephropathy, microalbuminuria, renal insufficiency, retinopathy, photocoagulation). Individual medications were identified based on chart review, whereas the total number of medications taken by an individual patient was derived from patient survey response. The mean number of prescribed medica- 
tions was 5.5, with a range from 0-20. To minimise analytic effects of the skewed distribution we categorised the number of medications into ordinal categories $(0-3,4$ $6,7-10$ and $>10$ medications). BMI data were missing for 218 patients $(24 \%)$; for these, BMI was imputed (as the mean of known BMI values) to avoid losing quality of life data from this subset. Seven hundred and seventy-two patients reported duration of diabetes; we imputed duration of diabetes by deriving the relationship of age, complications and insulin use to diabetes duration in those 772 patients, and using that formula to predict diabetes duration in the remainder. The mean duration $( \pm \mathrm{SD})$ was $9.6 \pm 7.6$ years before imputation and $9.6 \pm 7.3$ years after imputation.

\section{Survey instruments}

Assessment of health utility Health-related quality of life is quantified by assigning a numeric utility between 0 and 1 to a given health state, where 0 represents the state of death and 1 represents optimal health. The Health Utilities Index Mark III (HUI-3) is a multi-attribute utility model developed to derive community perspective health utilities (i.e. the general population's assessment of the health-related quality of life associated with a particular disease or condition) from patient responses to a questionnaire. The HUI-3 is simpler to administer and complete than other utility assessment methods, such as the standard gamble or time trade-off [16-18]. In the HUI-3, patients answer 15 questions about their level of function to define eight health domains (vision, hearing, speech, ambulation, dexterity, emotion, cognition and pain), each with five or six levels of impairment, ranging from none to severe impairment $[13,15]$. Survey responses are coded into one level on each domain, corresponding to the preferences (i.e. utilities) of a reference population [14]. These values are then combined by multiplying weighted domain levels $(W)$ according to the formula determined by validation of the HUI-3 model $(1.371 \times W 1 \times W 2 \times W 3 \times W 4 \times W 5 \times W 6 \times W 7 \times W 8)-0.371)$ to derive a composite overall health utility score anchored between 0 and 1 [19], with negative values representing health states worse than death. Scores in individual domains determine the overall utility score, and inspection of the individual domains suggests those that make the greatest contribution to low utility. According to the validation of the HUI-3, increments of 0.03 unit of health utility represent a clinically significant difference in quality of life [20]; consequently, results have been rounded to the second decimal place in this analysis. The population distribution of health utility is typically left-skewed, with a small number of patients with very low quality of life. In addition, the HUI may be overweighted to the low end of the scale relative to the standard gamble method of determining health utility $[17,21]$. Taking advantage of internal redundancy within the HUI-3 questionnaire, we used a validated method of inspection and logical deduction to impute HUI-3 data for 54 participants missing complete data on the HUI-3 [22].
The mean utility $( \pm \mathrm{SD}$ ) was $0.61 \pm 0.32$ before and $0.60 \pm 0.32$ after imputation.

Assessment of depression We used the validated 10-item Harvard Department of Psychiatry/National Depression Screening Day Scale (HANDS) to assess depression. This scale is scored from $0-3$ per item; a score of nine or greater has a $95 \%$ sensitivity for clinically significant levels of depression diagnosed according to the Diagnostic and Statistical Manual of Mental Disorders, Fourth Edition (DSM-IV), and confirmed by structured clinical interview diagnosis [23]. While the questions on the HUI and the HANDS are different, the HUI queries pain and emotion, which are related to the symptoms of depression queried by the HANDS. To check for criterion contamination (i.e. overlap between the HANDS and HUI questionnaires on constructs related to mood), we repeated analyses of effects of depression using diagnosed depression derived from a problem list diagnosis or prescription of antidepressant medication.

\section{Statistical analysis}

We reported descriptive statistics with standard deviations. Because the estimates of health utility were left-skewed and because the Health Utilities Index tends to underestimate utility values derived from standard gambles, the median, rather than mean, values of utility associated with individual demographic and clinical covariates are reported. Wilcoxon-derived $p$ values are reported for the difference in health utility between groups with and without the specified demographic and clinical covariates. The non-parametric Wilcoxon $p$ values did not differ from $p$ values derived by $t$ tests, which assume underlying normality.

Despite the mild skew of health utilities, ordinary least squares and other multiple regression techniques that assume normality are robust to this problem and have been used in multiple publications using the HUI [24, 25]. We therefore used general linear modelling to determine the least-squares means of multi-category variables and in multivariable models. We forced age and sex into multivariable general linear models to determine the independent correlates of health-related quality of life using variables that were significant in univariate analysis. Sociodemographic variables that were not significant in multivariable models were dropped from the models. The normal probability plots of the residuals of the multivariable models revealed that the residuals were normally distributed. Because depression was such a strong predictor of quality of life, first-order interactions between depression and sex, heart failure, microvascular complications, low educational level, and the presence of severe obesity were tested. We used a significance level of 0.05 for exclusion of variables from the model. Analyses were conducted using the SAS statistical system (SAS Institute, Cary, NC, USA). 


\section{Results}

Overall, patient health-related quality of life was moderately high. The mean utility of the group was $0.60 \pm 0.32$, with a median of 0.70 and an interquartile range of $0.39-$ 0.88 . The 90 th percentile was 0.97 , but the 10 th percentile was 0.07 . The distributions of the individual HUI-3 domains (Table 1a) revealed that emotion, pain and impaired vision were the predominant drivers of low overall health utility in these patients. Overall health utility (derived from community measures) correlated well with patient self-reported health (Table 1b).

The demographic and clinical characteristics of the study population, along with health-related quality of life, measured in utility, are shown in Table 2. In univariate analysis, quality of life differed by sex, race, level of education and employment status, but not by clinic site. For instance, the median estimate of quality of life among women was 0.63 , and among men, $0.75(p<0.0001)$. The patients were elderly, and most were overweight or obese. One-third had CHD, one-half had microvascular complications, and one-fifth had depression. Patients with depression had the lowest healthrelated quality of life (median utility 0.28 vs 0.69 for those without depression, $p<0.0001$ ). The health-related quality of life was reduced in patients with CHD, stroke, microvascular complications, heart failure, chronic obstructive pulmonary disease (COPD) or depression, but not in those with hypertension, hyperlipidaemia, elevated blood pressure, current smoking or obesity, compared with patients without these conditions.

Table 1 Mean and median utilities according to HUI-3 domains and self-reported health status in 909 patients with type 2 diabetes

\begin{tabular}{|c|c|c|c|c|}
\hline \multirow{2}{*}{\multicolumn{3}{|c|}{ Health utilities index domain ${ }^{a}$}} & \multicolumn{2}{|c|}{ Domain utility } \\
\hline & & & Mean & Median \\
\hline Vision & & & 0.91 & 0.95 \\
\hline Hearing & & & 0.88 & 1.00 \\
\hline Speech & & & 0.98 & 1.00 \\
\hline Ambulation & & & 0.87 & 1.00 \\
\hline Dexterity & & & 0.94 & 1.00 \\
\hline Emotion & & & 0.88 & 0.91 \\
\hline Cognition & & & 0.90 & 1.00 \\
\hline Pain & & & 0.82 & 0.92 \\
\hline \multicolumn{3}{|l|}{ Self-reported health status } & \multicolumn{2}{|c|}{ Overall utility } \\
\hline & Number $^{\mathrm{b}}$ & Percentage & Mean & Median \\
\hline Excellent & 116 & 22.0 & 0.85 & 0.92 \\
\hline Very good & 268 & 29.9 & 0.75 & 0.79 \\
\hline Good & 309 & 34.5 & 0.58 & 0.62 \\
\hline Fair & 163 & 18.2 & 0.37 & 0.36 \\
\hline Poor & 40 & 4.5 & 0.08 & -0.04 \\
\hline
\end{tabular}

${ }^{a}$ The overall health utility is a function of the component weighted levels of function on each of the domains according to the following formula determined by the initial validation of the HUI-3: $(1.371 \times W 1 \times W 2 \times W 3 \times W 4 \times W 5 \times W 6 \times W 7 \times W 8)-0.371$

${ }^{b} n=896$ due to missing responses to the question on self-reported health status
Most patients were prescribed multiple medications, and about one-quarter were prescribed insulin with or without an oral hypoglycaemic. Increasing number of medications, insulin use and duration of diabetes were associated with decreased health utility in univariate analysis. This was an obese and elderly cohort (mean BMI was $31.3 \pm 6.7 \mathrm{~kg} / \mathrm{m}^{2}$ ). Metabolic and cardiac risk factor control was fair, with mean values of $7.45 \pm 1.46 \%$ for $\mathrm{HbA}_{1 \mathrm{c}}, 4.4 \pm 0.9 \mathrm{mmol} / 1$ $(173 \pm 36.9 \mathrm{mg} / \mathrm{dl})$ for total cholesterol, $1.2 \pm 0.3 \mathrm{mmol} / \mathrm{l}$ $(44.5 \pm 13.4 \mathrm{mg} / \mathrm{dl})$ for $\mathrm{HDL}$ and $2.5 \pm 0.8 \mathrm{mmol} / 1(94.7 \pm$ $29.7 \mathrm{mg} / \mathrm{dl}$ ) for LDL; $58 \%$ of patients with blood pressure less than or equal to $130 / 80 \mathrm{mmHg}$. Utility was not correlated with level of glycaemic control or level of cholesterol $(p>0.3)$.

The age- and sex-adjusted decrements in health-related quality of life associated with specific clinical variables and multivariable-adjusted median utilities are shown in Table 3. Table 3a shows the decrease in utility associated with each variable, adjusted only for age and sex. For example, microvascular complications alone were associated with a utility penalty of 0.12 . After adjusting for the socioeconomic variables, multiple comorbidities and insulin use, shown in Table $3 b$, adjusted for all of the listed comorbidities and duration of diabetes, the penalty associated with comorbidities such as microvascular complications, heart failure, COPD and depression diminished to some degree, while CHD, stroke and insulin use were no longer independently associated with impairments in health-related quality of life. In the final multivariable model (Table 3c), microvascular complications, heart failure and number of medications were statistically and clinically significant correlates of decrement in overall health-related quality of life, but the presence of depression was the strongest correlate, decreasing utility by 0.37 when present. Model results were similar using depression identified by chart diagnosis rather than HANDS screen: the utility decrement associated with diagnosed depression was $0.14 \pm 0.02(p<0.0001)$. Model results were also similar when microvascular complications that were less likely to be symptomatic were excluded from the analysis. After including number of medications in the model, CHD, COPD, stroke, insulin and duration of diabetes were no longer significant correlates of health-related quality of life, though the $r^{2}$ value (proportion of variance explained by the model) was not substantially improved. First-order interactions between depression and sex, heart failure, complications and educational level were not significant ( $p>0.2$ for all).

\section{Discussion}

In this large, primary care clinic-based survey, we found that in the presence of specific comorbidities and complications, type 2 diabetes is associated with substantial decrements in health-related quality of life. Microvascular complications, heart failure, depression and a high number of medications were each strong independent correlates of decreased quality of life. Whereas a primary 
Table 2 Median utilities of 909 primary care patients with type 2 diabetes according to demographic and clinical characteristics

\begin{tabular}{|c|c|c|c|c|}
\hline & $\begin{array}{l}\text { Number } \\
\text { with } \\
\text { specified } \\
\text { characteristic }\end{array}$ & Percentage & $\begin{array}{l}\text { Median } \\
\text { utility for } \\
\text { group with } \\
\text { specified } \\
\text { characteristic }\end{array}$ & $p$ value $^{\mathrm{a}}$ \\
\hline Sample median & 909 & & 0.70 & \\
\hline \multicolumn{5}{|l|}{ Demographic characteristic } \\
\hline \multicolumn{5}{|l|}{ Sex } \\
\hline Female & 444 & 49 & 0.63 & \\
\hline Male & 465 & 51 & 0.75 & $<0.0001$ \\
\hline \multicolumn{5}{|l|}{ Age, years (mean $66.3 \pm 12.5$ ) } \\
\hline$<50$ & 90 & 10 & 0.61 & \\
\hline $50-59$ & 181 & 20 & 0.62 & \\
\hline $60-69$ & 233 & 26 & 0.64 & \\
\hline $70-79$ & 274 & 30 & 0.60 & \\
\hline$\geq 80$ & 131 & 14 & 0.54 & 0.08 \\
\hline \multicolumn{5}{|l|}{ Race } \\
\hline Non-Hispanic, white & 744 & 83 & 0.69 & \\
\hline Non-white & 153 & 17 & 0.75 & 0.1 \\
\hline \multicolumn{5}{|l|}{ Education } \\
\hline Less than high school diploma & 201 & 22 & 0.55 & \\
\hline $\begin{array}{l}\text { High school diploma, its equivalent, } \\
\text { or some time at college }\end{array}$ & 496 & 55 & 0.70 & \\
\hline Four years of college or advanced degree & 205 & 23 & 0.77 & $<0.0001$ \\
\hline \multicolumn{5}{|l|}{ Employment } \\
\hline Full-time & 195 & 21 & 0.85 & \\
\hline Part-time & 60 & 7 & 0.72 & \\
\hline Unemployed & 118 & 13 & 0.42 & \\
\hline Retired & 536 & 59 & 0.67 & $<0.0001$ \\
\hline \multicolumn{5}{|l|}{ Marital status } \\
\hline Married/living with partner & 477 & 53 & 0.74 & \\
\hline Single & 116 & 13 & 0.70 & \\
\hline Divorced/separated/widowed & 306 & 34 & 0.64 & $<0.0001$ \\
\hline \multicolumn{5}{|l|}{ Clinic site } \\
\hline Hospital-based & 550 & 61 & 0.70 & \\
\hline Community health centre & 359 & 40 & 0.70 & 0.9 \\
\hline \multicolumn{5}{|l|}{ Clinical characteristic } \\
\hline No complications ${ }^{\mathrm{b}}$ & 217 & 24 & 0.84 & $<0.0001$ \\
\hline $\mathrm{CHD}$ & 316 & 35 & 0.58 & $<0.0001$ \\
\hline Stroke & 148 & 16 & 0.56 & 0.004 \\
\hline Peripheral vascular disease & 130 & 14 & 0.58 & 0.001 \\
\hline Microvascular complications & 441 & 49 & 0.61 & $<0.0001$ \\
\hline Symptomatic microvascular complications & 370 & 41 & 0.61 & $<0.0001$ \\
\hline Hypertension & 811 & 89 & 0.69 & 0.3 \\
\hline Heart failure & 116 & 13 & 0.43 & $<0.0001$ \\
\hline Hyperlipidaemia & 684 & 75 & 0.70 & 0.5 \\
\hline COPD & 156 & 17 & 0.56 & $<0.0001$ \\
\hline Smoker & 109 & 12 & 0.61 & 0.1 \\
\hline \multicolumn{5}{|l|}{ BMI, $\mathrm{kg} / \mathrm{m}^{2}($ mean $31 \pm 7$ ) } \\
\hline Normal $(<25)$ & 150 & 17 & 0.64 & \\
\hline Overweight (25-29.9) & 286 & 32 & 0.73 & \\
\hline Obese $(\geq 30)$ & 453 & 50 & 0.68 & 0.02 \\
\hline Depression $^{\mathrm{c}}$ & 156 & 13 & 0.28 & $<0.0001$ \\
\hline \multicolumn{5}{|l|}{ Number of medications } \\
\hline $0-3$ & 201 & 23 & 0.79 & \\
\hline $4-6$ & 322 & 37 & 0.75 & \\
\hline
\end{tabular}

${ }^{\mathrm{a}}$ Wilcoxon $p$ value for difference in median utility between groups with and without the specified characteristic ${ }^{\mathrm{b}}$ Patients without coronary artery disease, stroke, microvascular complications, heart failure, COPD or depression ${ }^{c} n=832$ due to missing data from HANDS screening instrument

\section{Sex}

Mal

Age, years (mean $66.3 \pm 12.5$ )

$<50$

$60-69$

$70-79$

Non-Hispanic, white

Less than high school diploma

High school diploma, its equivalent,

Four years of college or advanced degree

mployment

Part-time

Unemployed

Marital status

Married/living with partner

Single

Clinic site

Hospital-based

Cinical characteristic

No complications ${ }^{\mathrm{b}}$

Stroke

Peripheral vascular disease

Hypertension

Hyperlipidaemia

BMI, $\mathrm{kg} / \mathrm{m}^{2}$ (mean $31 \pm 7$ )

Normal $(<25)$

Overweight (25-29.9)

Number of medications

322
Number

specified

characteristic
0.70

0.63

0.61

0.64

0.69

0.55

0.77

0.42

0.74

0.70

0.70

0.70

0.84

$<0.0001$

0.004

0.001

0.0001

0.5

0.000

0.02

$<0.0001$ 
Table 2 (continued)

\begin{tabular}{|c|c|c|c|c|}
\hline & $\begin{array}{l}\text { Number } \\
\text { with } \\
\text { specified } \\
\text { characteristic }\end{array}$ & Percentage & $\begin{array}{l}\text { Median } \\
\text { utility for } \\
\text { group with } \\
\text { specified } \\
\text { characteristic }\end{array}$ & $p$ value $^{\mathrm{a}}$ \\
\hline $7-10$ & 243 & 28 & 0.54 & \\
\hline$>10$ & 113 & 13 & 0.40 & $<0.0001$ \\
\hline \multicolumn{5}{|l|}{ Hypoglycaemic medications } \\
\hline Oral hypoglycaemic only & 429 & 47 & 0.72 & 0.2 \\
\hline Insulin with or without oral hypoglycaemic & 242 & 27 & 0.56 & $<0.0001$ \\
\hline Metformin & 174 & 19 & 0.65 & 0.05 \\
\hline Sulfonylurea & 364 & 40 & 0.60 & 0.47 \\
\hline \multicolumn{5}{|l|}{ Duration of diabetes } \\
\hline$\leq 3$ years & 181 & 20 & 0.65 & \\
\hline $4-6$ years & 191 & 21 & 0.66 & \\
\hline $7-10$ years & 217 & 24 & 0.63 & \\
\hline$\geq 11$ years & 320 & 35 & 0.52 & $<0.0001$ \\
\hline Blood pressure $\leq 130 / 80 \mathrm{mmHg}$ & 524 & 58 & 0.59 & 0.06 \\
\hline
\end{tabular}

care patient with type 2 diabetes who lacked any of the variables specified in the model had a predicted overall health utility of $0.96 \pm 0.05$, the presence of microvascular complications reduced predicted utility to 0.91 , and if such a patient also had heart failure and depression, predicted utility dropped to 0.44 . These estimates represent an improvement over prior estimates because of the setting and because of the degree of clinical detail, which allowed us to elucidate the determinants of quality of life.

The study was conducted in a usual care setting, had a very high response rate, and is one of the largest studies of health utility in primary care patients with type 2 diabetes in the US. This is also the first study to provide utility estimates adjusted for the presence of depression in addition to other demographic and clinical variables. While many studies have found that conditions such as hypertension, obesity and use of insulin $[1,9,10]$ were correlated with decreased quality of life, we did not find these conditions to be significant after controlling for more symptomatic conditions and overall treatment intensity. Notably, other asymptomatic conditions, such as hyperlipidaemia, were not associated with decreased quality of life in this cohort. Poor glycaemic control, which is frequently symptomatic, was also not associated with decreased quality of life, consistent with recent findings in another population [26]. Symptomatic conditions such as heart failure [8] and macrovascular or microvascular complications [1, 8, 9, 27] of diabetes have consistently been shown to be associated with decreased health-related quality of life. Female gender $[4,9,27]$ and indicators of low socioeconomic status $[4,27]$ have been identified as independent correlates of decreased health-related quality of life in other studies of patients with diabetes and in the general population [28]. The association of some of these conditions with decreased quality of life appears to be explained, in part, by the presence of complications and concurrent depression.

In the multivariable models, the number of medications was a strong correlate of decreased utility. As in other studies of health-related quality of life, people who take a large number of medications rate their health as poorer than those who do not [18]. This correlation may be due to reverse causality: sicker patients are prescribed more medications, but taking a large number of medications may decrease quality of life. A negative impact of medication on quality of life might be mediated by the effect of medication-taking behaviour, expense or side effects. The number of medications taken represents treatment intensity and correlates with severity of comorbid disease. After including this variable in model 3c (Table 3), some comorbidities and duration of diabetes were no longer significant, but the more symptomatic comorbidities remained significant correlates of health-related quality of life; the overall explanatory power of the model was not substantially improved. This suggests that while the number of medications is conceptually related to severity of comorbid disease, it is not completely collinear. We suspect that the strong correlation between number of medications and quality of life is partially related to severity of disease, but decreased quality of life may also be related to the burden of the medication regimen itself.

Insulin was not a statistically significant correlate of decreased quality of life in multivariable regression $(p=0.7$ after controlling for duration of diabetes), but was signif- 
Table 3 Relationship of clinical variables to health-related quality of life in primary care patients with type 2 diabetes

\begin{tabular}{|c|c|c|c|c|c|c|c|c|c|}
\hline & \multicolumn{3}{|c|}{$\begin{array}{l}\text { Model A: age- and sex-adjusted } \\
\text { associations of individual variables } \\
\text { with HRQOL }\end{array}$} & \multicolumn{3}{|c|}{$\begin{array}{l}\text { Model B: multiple regression with } \\
\text { HRQOL, not including number of } \\
\text { medications. } n=827 ; R^{2}=0.36\end{array}$} & \multicolumn{3}{|c|}{$\begin{array}{l}\text { Model C: multiple regression with } \\
\text { HRQOL, including number of } \\
\text { medications. } n=808 ; R^{2}=0.39\end{array}$} \\
\hline Intercept & - & & & 0.93 & 0.06 & & 0.96 & 0.05 & \\
\hline $\begin{array}{l}\text { Education }<12 \text { th } \\
\text { grade }^{\mathrm{a}}\end{array}$ & -0.16 & 0.03 & $<0.0001$ & -0.08 & 0.03 & 0.003 & -0.09 & 0.03 & 0.0007 \\
\hline $\begin{array}{l}\text { Microvascular } \\
\text { complications }\end{array}$ & -0.12 & 0.02 & $<0.0001$ & -0.06 & 0.02 & 0.002 & -0.05 & 0.02 & 0.02 \\
\hline Heart failure & -0.24 & 0.03 & $<0.0001$ & -0.13 & 0.03 & $<0.0001$ & -0.10 & 0.03 & 0.001 \\
\hline Insulin & -0.10 & 0.03 & $<0.0001$ & -0.009 & 0.02 & 0.7 & -0.006 & 0.02 & 0.8 \\
\hline 7-10 medications & -0.17 & 0.03 & $<0.0001^{\mathrm{c}}$ & - & & & -0.10 & 0.02 & 0.0003 \\
\hline$>10$ medications & -0.29 & 0.04 & $<0.0001^{\mathrm{c}}$ & - & & & -0.19 & 0.03 & $<0.0001$ \\
\hline $\begin{array}{l}\text { Diabetes duration } \\
>10 \text { years }\end{array}$ & -0.12 & 0.03 & $<0.0001^{\mathrm{d}}$ & -0.06 & 0.03 & 0.03 & -0.04 & 0.03 & 0.1 \\
\hline
\end{tabular}

Interpretation of age- and sex-adjusted models: the parameter represents the utility penalty incurred by an age- and sex-standardised patient with the specified clinical variable. For instance, the model estimates that heart failure in a patient with type 2 diabetes would decrease utility by 0.24

Interpretation of the multivariable models: the intercept represents the overall health utility of an individual (male) of the mean age of the population ( 66 years) without any of the conditions specified in the model. The parameter estimates represent the utility penalty incurred by an individual controlling for all other covariates in the model. For each condition, subtract the parameter estimate of the significant variable to determine health utility. For example, in model $\mathrm{C}$, a patient with type 2 diabetes and heart failure alone would have a utility of 0.86 ; a patient with type 2 diabetes, heart failure, and depression would have a utility of 0.52

$H R Q O L$ Health-related quality of life

${ }^{\mathrm{a}} n=902 ;{ }^{\mathrm{b}} n=832$; ${ }^{\mathrm{c}} n=879$, compared with $0-3$ medications; ${ }^{\mathrm{d}} n=909$, compared with $<3$ years

icant in univariate analysis, and many studies have found that insulin use in association with complications is associated with decreased quality of life $[9,10]$. The purported relationship of health-related quality of life to insulin use presents another dilemma of reverse causality. This has led some authors to suggest that delaying insulin therapy will improve quality of life [9], but these studies have not controlled for measures of severity of disease that are correlated with insulin use. In another large US population study in which multiple comorbidities were controlled for, insulin was not a significant predictor of decreased quality of life [8]. In keeping with the latter study and the UKPDS [1], we found that symptomatic conditions such as heart failure and microvascular complications were far more significant correlates of lower quality of life than unsymptomatic conditions. We conclude from this analysis that insulin has served as a proxy for sicker patients in studies that have not been able to control for these variables.

Depression is a well-recognised determinant of quality of life in diabetes $[4,10]$. However, most prior estimates of health utility in type 2 diabetes have failed to account for depression, thus producing biased estimates of health utility. Patients with depression rate their quality of life lower than those with other chronic diseases [29]. Our models show that depression decreases health utility by $0.37-0.38$, an estimate that is consistent with prior studies in other disease models $[30,31]$. After including depression in our models, parameter estimates of the degree of disutility associated with heart failure and complications decreased, producing a less biased estimate of the independent effect of these comorbidities. Given that patients with depression are prone to rate their overall health as very poor, and that the prevalence of depression in patients with diabetes is high and correlated with comorbidities [32], our results highlight the importance of accounting for depression in any analysis of healthrelated quality of life in diabetes.

Estimates of mean utility in diabetes have ranged widely, from $0.69 \pm 0.39$ in a study of 372 rural patients with type 2 diabetes in Alberta, Canada [12], to a mean of 0.89-0.90 in population surveys in Ontario [11,33]. Our sample mean of $0.60 \pm 0.32$ and median of 0.70 is in line with, but slightly lower than, results from other studies and represents a large 
US primary care patient population. It may be on the low end of estimates due to the severity of illness in this population or due to the formula used to calculate the HUI3 , which is overweighted on the low end of the scale. The relationship between the absolute numbers and patient status can be gauged by inspecting the mean utilities associated with self-reported health status (Table 1b).

These estimates are comparable to those found in other patients with chronic illness surveyed with the HUI-3. For example, 103 British patients undergoing elective arthroplasty had a mean utility of 0.63 [21], 87 patients with claudication pre-revascularisation had a mean utility of 0.66 [34], and 679 patients with Alzheimer's disease and their caregivers had mean utilities of 0.22 and 0.87 , respectively [35]. Our results are lower than those found in a recent population survey of 4,048 US adults (mean age 45 ), who had a mean utility of $0.81 \pm 0.01$ on the HUI-3 [28].

In these studies and in ours, global utilities capture the impact of many of the evanescent symptoms associated with a given disease which can be hard to measure. For example, while symptoms of hyperglycaemia and hypoglycaemia may be transiently symptomatic and thus hard to measure as a specific comorbidity, the global utility can capture the sense of diminished well-being that may accompany such symptoms. It is also important to recognise that numeric utility estimates will vary according to population; consequently, sensitivity analysis should be employed when using utility estimates in cost-effectiveness models.

There are several limitations of our analysis. The lack of racial and ethnic heterogeneity restricted our ability to analyse these groups individually. Moreover, ethnic minorities and non-English speakers were less likely to participate in the study. Non-participants also had worse glycaemic and blood pressure control, but were more likely to be on a blood pressure medication or aspirin; the prevalence of depression and antidepressants were similar. Non-participants are likely to represent a sicker group who were nonetheless being treated for comorbidities. It is difficult to anticipate how these results would apply to that cohort, but it is likely that health utility would be lower in sicker patients who either could not participate or whose primary care doctors excluded them from the study, so the present results may underestimate the true impact of diabetes on quality of life. HUI-3 utilities represent community perspectives and may differ from those elicited directly from patients (for instance, using a standard gamble assessment), who may rate their quality of life higher than a community observer (with the exception of subjects with depression). While this should not affect the correlates of impaired quality of life, it is likely to affect the absolute utility values. Community-perspective utilities are useful for cost-effectiveness analysis and policy decisions, but patient utilities may be more useful for clinical decision-making [16]. In addition, an instrument that uses 15 questions to measure eight domains will necessarily fail to capture many disease-specific symptoms; while a generic instrument has the advantage of generalisability and comparability across studies, it will certainly miss many disease-specific subtleties.
In summary, in addition to providing more precise estimates of utilities that may be used in cost-effectiveness analysis, this study of responses to the HUI-3 questionnaire by 909 primary care patients with type 2 diabetes revealed important correlates of health-related quality of life. This is the first study to find that a patient with type 2 diabetes has minimal impairment in health-related quality of life in the absence of socioeconomic hardships, disease complications, or depression. Symptomatic conditions such as heart failure, microvascular complications, and treatment intensity (which may be a proxy for severity of disease) correlated with impaired quality of life. Based on these results, treating depression and preventing symptomatic complications have the greatest potential to improve health-related quality of life in patients with type 2 diabetes.

Acknowledgements This study was supported by a grant from Pfizer, with additional support from Aventis and the Massachusetts General Hospital Clinical Research Program. J. B. Meigs is supported by an American Diabetes Association Career Development Award. R. W. Grant is supported by a Career Development Award from the National Institutes of Health (NIDDK DK067452).

Duality of interest We declare that we have no conflict of interest.

\section{References}

1. UK Prospective Diabetes Study Group (1999) Quality of life in type 2 diabetic patients is affected by complications but not by intensive policies to improve blood glucose or blood pressure control (UKPDS 37). Diabetes Care 22:1125-1136

2. Vijan S, Hayward RA, Ronis DL, Hofer TP (2005) The burden of diabetes therapy: implications for the design of effective patient-centered treatment regimens. J Gen Intern Med 20:479-482

3. Polonsky WH (2002) Emotional and quality-of-life aspects of diabetes management. Curr Diab Rep 2:153-159

4. Goldney RD, Phillips PJ, Fisher LJ, Wilson DH (2004) Diabetes, depression, and quality of life: a population study. Diabetes Care 27:1066-1070

5. Bourdel-Marchasson I, Dubroca B, Manciet G, Decamps A, Emeriau JP, Dartigues JF (1997) Prevalence of diabetes and effect on quality of life in older French living in the community: the PAQUID Epidemiological Survey. J Am Geriatr Soc 45:295-301

6. Jacobson AM, de Groot M, Samson JA (1997) The effects of psychiatric disorders and symptoms on quality of life in patients with type I and type II diabetes mellitus. Qual Life Res 6:11-20

7. Kilbourne AM, Reynolds CF, Good CB 3rd, Sereika SM, Justice AC, Fine MJ (2005) How does depression influence diabetes medication adherence in older patients? Am J Geriatr Psychiatry 13:202-210

8. Coffey JT, Brandle M, Zhou H et al (2002) Valuing healthrelated quality of life in diabetes. Diabetes Care 25:2238-2243

9. Redekop WK, Koopmanschap MA, Stolk RP, Rutten GE, Wolffenbuttel BH, Niessen LW (2002) Health-related quality of life and treatment satisfaction in Dutch patients with type 2 diabetes. Diabetes Care 25:458-463

10. Brown GC, Brown MM, Sharma S, Brown H, Gozum M, Denton P (2000) Quality of life associated with diabetes mellitus in an adult population. J Diabetes Complications $14: 18-24$ 
11. Manuel DG, Schultz SE (2004) Health-related quality of life and health-adjusted life expectancy of people with diabetes in Ontario, Canada, 1996-1997. Diabetes Care 27:407-414

12. Maddigan SL, Feeny DH, Johnson J (2003) A comparison of the health utilities indices Mark 2 and Mark 3 in type 2 diabetes. Med Decis Making 23:489-501

13. Horsman J, Furlong W, Feeny D, Torrance G (2003) The health utilities index (HUI): concepts, measurement properties and applications. Health Qual Life Outcomes 1:54

14. Feeny D, Furlong W, Torrance GW et al (2002) Multiattribute and single-attribute utility functions for the health utilities index Mark 3 system. Med Care 40:113-128

15. Furlong WJ, Feeny DH, Torrance GW (2000) Health utilities index procedures manual. Health Utilities, Dundas, Ontario, Canada

16. Gold MR, Patrick DL, Torrance GW et al (1996) Identifying and valuing outcomes. In: Gold MR, Siegel JE, Russell LB, Weinstein MC (eds) Cost-effectiveness in health and medicine. Oxford University Press, New York, pp 83-134

17. Feeny D, Furlong W, Saigal S, Sun J (2004) Comparing directly measured standard gamble scores to HUI2 and HUI3 utility scores: group- and individual-level comparisons. Soc Sci Med 58:799-809

18. Stewart ST, Woodward RM, Cutler DM (2005) A proposed method for monitoring U.S. population health: linking symptoms, impairments, chronic conditions, and health ratings. In: NBER Working Paper Series. National Bureau of Economic Research, Cambridge, Massachusetts, USA

19. Brazier J, Deverill M, Green C, Harper R, Booth A (1999) A review of the use of health status measures in economic evaluation. Health Technol Assess 3:1-164

20. Drummond M (2001) Introducing economic and quality of life measurements into clinical studies. Ann Med 33:344-349

21. Feeny D, Blanchard C, Mahon JL et al (2003) Comparing community-preference-based and direct standard gamble utility scores: evidence from elective total hip arthroplasty. Int $\mathbf{J}$ Technol Assess Health Care 19:362-372

22. Naeim A, Keeler E, Mangione C (2005) Options for handling missing data in the health utilities index Mark 3. Med Decis Making 25:186-198

23. Baer L, Jacobs DG, Meszler-Reizes J et al (2000) Development of a brief screening instrument: the HANDS. Psychother Psychosom 69:35-41
24. Grootendorst P, Feeny D, Furlong W (2000) Health utilities index Mark 3: evidence of construct validity for stroke and arthritis in a population health survey. Med Care 38:290-299

25. Kopec JA, Williams JI, To T, Austin PC (2000) Measuring population health: correlates of the health utilities index among English and French Canadians. Can J Public Health 91:465-470

26. Maddigan SL, Feeny DH, Johnson JA (2004) Construct validity of the RAND-12 and health utilities index Mark 2 and 3 in type 2 diabetes. Qual Life Res 13:435-448

27. Glasgow RE, Ruggiero L, Eakin EG, Dryfoos J, Chobanian L (1997) Quality of life and associated characteristics in a large national sample of adults with diabetes. Diabetes Care $20: 562-567$

28. Luo N, Johnson JA, Shaw JW, Feeny D, Coons SJ (2005) Selfreported health status of the general adult U.S. population as assessed by the EQ-5D and Health Utilities Index. Med Care 43:1078-1086

29. Wells KB, Sherbourne CD (1999) Functioning and utility for current health of patients with depression or chronic medical conditions in managed, primary care practices. Arch Gen Psychiatry 56:897-904

30. Revicki DA, Wood M (1998) Patient-assigned health state utilities for depression-related outcomes: differences by depression severity and antidepressant medications. J Affect Disord 48:25-36

31. Ruo B, Rumsfeld JS, Hlatky MA, Liu H, Browner WS, Whooley MA (2003) Depressive symptoms and health-related quality of life: the Heart and Soul Study. JAMA 290:215-221

32. Engum A, Mykletun A, Midthjell K, Holen A, Dahl AA (2005) Depression and diabetes: a large population-based study of sociodemographic, lifestyle, and clinical factors associated with depression in type 1 and type 2 diabetes. Diabetes Care 28:1904-1909

33. Maddigan SL, Feeny DH, Johnson JA (2005) Health-related quality of life deficits associated with diabetes and comorbidities in a Canadian National Population Health Survey. Qual Life Res 14:1311-1320

34. Bosch JL, Halpern EF, Gazelle GS (2002) Comparison of preference-based utilities of the Short-Form 36 health survey and health utilities index before and after treatment of patients with intermittent claudication. Med Decis Making 22:403-409

35. Neumann PJ, Sandberg EA, Araki SS, Kuntz KM, Feeny D, Weinstein MC (2000) A comparison of HUI2 and HUI3 utility scores in Alzheimer's disease. Med Decis Making 20:413-422 